\title{
COMPARING SIMULATION ALTERNATIVES BASED ON QUALITY EXPECTATIONS
}

\author{
Joshua Bosire \\ Shengyong Wang \\ Department of Systems Science \\ and Industrial Engineering \\ P.O. Box 6000 \\ Binghamton University (SUNY) \\ Binghamton, NY 13902, USA
}

Tejas Gandhi

Management Engineering Department

50 Lake Center Drive, Suite 120

Virtua Health

Marlton, NJ 08053, USA
Krishnaswami Srihari

Department of Systems Science and Industrial Engineering

P.O. Box 6000

Binghamton University (SUNY)

Binghamton, NY 13902, USA

\begin{abstract}
Computed Tomography (CT) is one of the fastest growing diagnostic imaging procedures. Rapid advances in imaging technologies in conjunction with their widening adoption are some of the issues that are compelling healthcare providers to restructure their systems as they seek to offer a higher quality of care to a growing volume of patients. This paper presents the application of simulation to facilitate the planning of a new CT facility for a hospital. The objective of the study was to evaluate how patient experience would be impacted by proposed design options. Waits for service were utilized as a parameter to quantify the patients' quality expectations, and hence the satisfaction derived from the healthcare services received. This study was also intended to clarify whether an additional CT-scan unit was a necessity to improving the patients' experience.
\end{abstract}

\section{INTRODUCTION}

Over the last few decades, simulation methodologies have progressed to become one of the leading experimentation techniques, especially when dealing with problems associated with uncertainty. As pointed out by Davis (1999), simulation analysis has been proven to be valuable in domains involving (1) establishing 'optimal' design settings for new or existing systems, (2) evaluating business scenarios to facilitate effective planning and management, and (3) the 'controlled' training of personnel, especially to enhance their psychomotor skills.

Modern hospitals and clinics are faced with escalating levels of competition in both domestic and global markets. At the same time, patients are increasingly demanding for a higher quality of healthcare services delivered at an equitable cost. Thus, healthcare organizations are increasingly pursuing efforts that will improve the efficiency and effectiveness of their patient flow dynamic
(Jun et al., 1999). This will facilitate the realization of streamlined care processes, thereby enabling the providers to improve the quality of care delivered as well as the productivity levels attained. To realize this, an increasing number of healthcare organizations are redesigning their existing facilities or planning for and designing new ones as a means to cater for the changing demand and patient flow dynamics. To a great extent, this has been facilitated by the adoption of various Industrial and Systems Engineering (ISE) techniques. Simulation is a key analysis methodology that has been widely adopted for similar endeavors (Jun et al., 1999; Ramakrishnan et al., 2004).

This research endeavor involved the adoption of discrete event simulation to facilitate the planning process for the CT-scan facilities of a new hospital. The study was part of a hospital planning and design project that was initiated to develop a structurally and functionally improved hospital to replace existing facilities. This was in accordance with the organization's strategic plan to improve the quality of care delivered by creating an enabling environment consisting of efficient and patient centered processes. The planning process entailed identifying an ideal location, establishing the need for various healthcare services and designing facilities that enhanced the quality, safety and productivity of the care delivery processes. The design of the various facilities involved identifying user and patient requirements, and subsequently translating them to design parameters that would be used to facilitate the development of an architectural plan for the new hospital. This study specifically focused on facilitating the design of a CT scan area that would cater for the changing demand dynamics, with $\mathrm{CT}$ procedures being expected to grow by about $60 \%$ within the next decade (sg2, 2007).

As part of the design process, a simulation study was initiated to evaluate the CT scan area with the aim of enabling decision making regarding (1) the number of CTscan units that would eventually be planned for, and (2) the 'optimal' positioning of these units on the facility lay- 
out. The study entailed an analysis of the CT examination processes, followed by a quantification of the impact of the location and capacity of the CT-scan units on the patients' quality expectations.

The remainder of this paper is structured as follows. Section 2 presents a statement of the study objectives, followed by a delineation of the study methodology in Section 3. Section 4 gives an overview of the CT examination processes, followed by Sections 5 and 6 which present the data collection and baseline modeling stages of the study. This is followed by an evaluation of the suggested design options in Section 7 and a summary of the study outcomes in Section 8.

\section{PROBLEM DEFINITION}

This study was part of the efforts that were focused on facilitating the design of a new hospital with enhanced structural and functional characteristics. The study was conducted to meet the objectives listed below.

1) Identify 'key process indicators' for the processes associated with CT-scan examinations.

2) Establish the performance of the baseline (existing) CT-scan processes.

3) Evaluate the impact of increasing the capacity of the CT-scan units.

4) Study the impact of the facility layout on the examination process - i.e. the difference in the performance measures for a "centralized" versus a "decentralized" CT-scan facility.

\section{STUDY METHODOLOGY}

To facilitate the realization of the established objectives, this study called upon various tools and techniques in the manner delineated below.

1) Process mapping and value-stream mapping techniques were utilized to study the current CT examination processes, commencing with the generation of a procedure order and ending with the completion of the CT procedure.

2) Modeling and simulation was identified as the ideal technique for analyzing the CT processes in order to achieve the study objectives. The choice was based on the understanding that simulation methodologies were best suited for evaluating "what-if" scenarios in situations with uncertainty and variability, similar to the CT-scan processes.

3) Observations were conducted and process data collected. The data was mathematically processed to yield probabilistic definitions of the examination process.

4) Simulation models were created to replicate the $\mathrm{CT}$ processes. These were verified and validated before being modified and used to study "what- if" scenarios that were formulated to test the various facets of the $\mathrm{CT}$-scan processes.

\section{THE CT EXAMINATION PROCESSES}

The CT-scan facility consisted of two imaging units which were utilized to examine Inpatients, Outpatients and patients from the emergency department (EDpatients). The first unit (M1) was a "sixty four-slice" scanner that was located within the emergency department (ED). The second unit (M2) was a "four-slice" scanner located some distance from the ED. M1 was preferred over M2 primarily because of its superior imaging capabilities, which enabled complex procedures (e.g. CT of the head/brain) to be performed. Consequently, M1 served the majority of the CT-scan needs whereas M2 was a back-up scanner that was also designated for longer procedures (e.g. breast biopsy).

Patient arrivals to the CT scan area occurred throughout the day for the entire week. Inpatient and ED-patient procedures were performed on a FIFO basis for all days of the week, albeit the ED-patient procedures often got precedence over Inpatient procedures - especially when they were flagged as 'urgent' (STAT). Outpatient procedures were always scheduled to be performed during the day-shift on all days of the week except Sunday. On arrival, all outpatients would be registered before being directed to the next available CT-scan unit. Outpatients would often queue in a waiting lounge while any ongoing procedures were completed. On the other hand, Inpatients were typically brought to the examination area by patienttransfer aides (transporters) while ED-patients were brought in by either a 'multi-skilled technician' (MST), a nurse (RN) or the CT technologist. The general flow of the $\mathrm{CT}$ processes is illustrated in Figure 1.

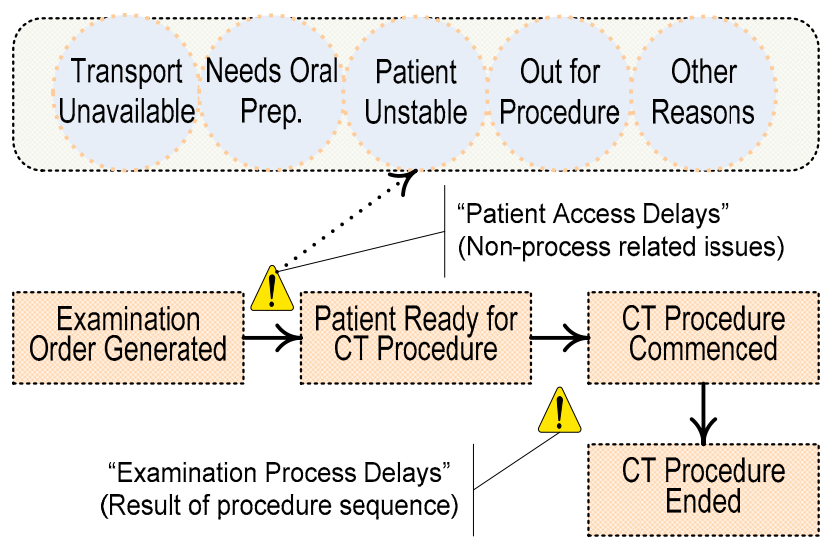

Figure 1: Illustration of the CT Examination Process.

Whenever a CT examination was requested, an "order-sheet" was generated and automatically printed out at M1. The order sheets were then queued and used to coor- 
dinate patient arrivals to the examination area. Typically, the orders were fulfilled using a FIFO discipline, with the exception of STAT orders from the ED. Once printed out, the order-sheet would be picked up and examined by a technologist to determine whether any pre-examination preparations were needed (e.g. the ingestion of oral contrast for abdominal scans). The technologist would then assign an "expected-arrival" time to the order based on the approximate time that would be required to finish the pre-examination preparations identified (if any). The orders were subsequently queued according to these "expected-arrival" times.

To fulfill the queued orders, a technologist would pick up the first order-sheet in queue and call the corresponding patient holding area to request that the patient be brought for examination. Typically the scheduled technologists worked concurrently, at times using both M1 and M2, to fulfill the queued orders. Thus, a request could be made to bring in the next patient (1) before completion of the current procedure, implying that more than one technologist was on duty, or (2) after completion of the current procedure, implying that only one technologist was on duty. This implies that patients would often wait (delay) for service if (1) the technologist and/ or imaging unit were busy when the next patient arrived - an 'examination process delay', or (2) the expected patient (Inpatient or ED-patient) was not ready for examination at the estimated time - a 'patient access delay'. These are the two forms of delay that this study primarily focused on. Figure 1 illustrates the various points in the examination process where these delays were encountered.

\section{DATA COLLECTION AND ANALYSIS}

To aid in modeling the behavior of the CT examination processes, system data was collected to capture key model inputs including the (1) time for which the CT procedures were ordered, (2) time when the procedures commenced, and (3) time when the patients departed the examination area. For the initial analysis, four months of data (representing the third quarter of 2006) was extracted from the Radiology Information System (RIS) and subjected to statistical scrutiny. For gauging purposes, this data was complemented by conducting time studies on the CT examination process. Eventually, the data was utilized to compute probabilistic input distributions for the simulation models of the CT examination process. This section elaborates the data collection and analysis stages of the simulation study.

\subsection{Data Gauging and 'Pre-processing'}

For initial analysis, the raw data from the RIS was utilized to extrapolate two measurements: (1) the intrinsic system delays (the 'patient access delays' shown in Figure 1) - time elapsed between order generation and completion of examination preparations, and (2) the service times time elapsed between commencement and completion of a CT procedure. The 'process delay' (time elapsed between the completion of examination preparations and the start of the procedure) was established as a 'key process indicator' (KPI) as it was purely process-related. The data was then stratified to summarize information pertaining to the different patient categories (IP, OP and ED) as well as the 'significant procedure' categories (biopsy \& nonbiopsy). Subsequently, discussions were conducted with the process experts to ascertain how well the RIS data described the actual system. It was revealed that the RIS data collection methodology was subjective and prone to error, yielding relatively skewed data that could not accurately describe the system operations. This view was supported by observations that were made at the CT-scan facilities.

To overcome these data challenges, the raw data from the RIS was subjected to pre-processing based on the 'gauge data' obtained from time studies and discussions with the process experts. The statistical summary for the 'total waits for service' was computed and compared to the 'gauge data'. Based on this, the raw data from the RIS was truncated and subsequently analyzed in MINITAB ${ }^{\circledR}$ to eliminate the potential outliers identified. A sample of the results of this pre-processing (cleaning), for the data associated with Inpatient wait times, is shown in Table 1. As illustrated, the raw data was highly skewed, containing both negative and extensively long wait times which were voided based on both time studies and expert opinions.

Table 1: Pre-processing for the RIS Raw Data.

\begin{tabular}{|l|r|r|r|r|}
\hline \multirow{3}{*}{$\begin{array}{c}\text { Comparative } \\
\text { Statistic }\end{array}$} & \multicolumn{3}{|c|}{ IP Wait Times (minutes) } \\
\cline { 2 - 5 } & $\begin{array}{c}\text { With Oral Contrast } \\
\text { Preparation }\end{array}$ & $\begin{array}{c}\text { No Oral Contrast } \\
\text { Preparation }\end{array}$ \\
\cline { 2 - 5 } & Raw Data & Processed & Raw Data & Processed \\
\hline Minimum & -1149 & 15 & -1310 & 15 \\
\hline Mean & 217.5 & 212.8 & 160.2 & 151.2 \\
\hline Mode & 0 & 100 & 0 & 16 \\
\hline Median & 189 & 198 & 86 & 111 \\
\hline Maximum & 2012 & 543 & 6039 & 543 \\
\hline St. Dev. & 284.9 & 111.8 & 295.5 & 124.4 \\
\hline Sample (n) & 376 & 303 & 1870 & 1458 \\
\hline
\end{tabular}

Similarly, the statistical summary for the 'service times' for both biopsy and non-biopsy procedures was computed from the raw data from the RIS. The nonbiopsy data was gauged against data collected from time studies (collected sample size: $n=18$ ) whereas the biopsy data was truncated based on the experts opinion and subsequently pre-processed. The resulting (cleaned) nonbiopsy data was eventually rejected as it could not yield 
'good fits' with any common theoretical probability distribution functions (the data was analyzed using Rockwell Arena's Input Analzyer ${ }^{\mathbb{B}}$ ). Consequently, the data obtained from the time studies was utilized to model the 'service times' for the non-biopsy procedures. Further details on the input modeling are presented in Section 5.2.

\subsection{Input Data Modeling}

As elaborated earlier, the model for the CT processes required three main data inputs: (1) arrival rates for different patient types on different days of the week, (2) access delays for all patient categories, and (3) service times for biopsy and non-biopsy procedure types. The demand for CT services (generation of orders) was presumed to represent an arrival pattern. To model these arrivals, the 'procedure request' times were first stratified according to the various patient categories and then analyzed for trends. For each patient category (IP, OP, ED), analysis was made based on both the hour of the day and the day of the week. Two-way ANOVA tests were then conducted to check for differences exhibited along these factors. The ANOVA findings are summarized in Table 2 whereas a sample of the trends exhibited in the demand for CT services is illustrated in Figure 2 (weekdays are presented by a solid line while weekends are shown as a broken-line). Consequently, the arrival patterns exhibiting significant differences were separately modeled as 'non-stationary Poisson processes' (Law and Kelton, 2003), with the arrival rates changing at each hour of the day.

Table 2: Modeling the Patient Arrivals.

\begin{tabular}{|c|l|}
\hline $\begin{array}{c}\text { PATIENT } \\
\text { TYPE }\end{array}$ & INFERENCES FROM ANOVA \\
\hline $\begin{array}{c}\text { Emergency } \\
\text { Room Patients } \\
\text { (ED) }\end{array}$ & $\begin{array}{l}\text { Different hourly arrival rates } \\
\text { arrivals rate for all days of the week } \\
\text { arrivals }\end{array}$ \\
\hline $\begin{array}{c}\text { Inpatients } \\
\text { (IP) }\end{array}$ & $\begin{array}{l}\text { Different hourly arrival rates } \\
\text { Rate of weekend arrivals significantly } \\
\text { different from that of the weekdays }\end{array}$ \\
\hline $\begin{array}{c}\text { Outpatients } \\
\text { (OP) }\end{array}$ & $\begin{array}{l}\text { Different hourly arrival rates } \\
\text { No significant difference in average } \\
\text { arrivals rate for all days of the week } \\
\text { No arrivals scheduled for Sundays }\end{array}$ \\
\hline
\end{tabular}

Since the 'patient access delays' could not be further stratified into their specific causative factors, they were classified into generic categories which were subsequently modeled as probability density functions (pdf). As shown in Table 3, patient access delays associated with each patient category were classified as either requiring or not-requiring oral contrast preparation. The pdf's associated with each of these delays were then established and subsequently utilized in developing the simulation model.
Finally, the time needed to conduct the CT-scan procedures (service time) was modeled based on whether the procedure was a biopsy or not. This distinction was based on the realization that biopsy procedures could last up to three hours whereas all the other procedures required at most 30 minutes to be completed. For the reasons elaborated in section 5.1 of this paper, the service times for the non-biopsy procedures were modeled as a Triangular distribution [TRIA $(5,10,30)$ ] while the service times for the biopsy procedures were established to follow a Log Normal distribution [LOGN $(85.87,0.39)$ ]. The Log Normal distribution was truncated to ensure that the associated service times did not exceed 3.5 hours, as this was established to be the longest duration that a biopsy procedure could last.

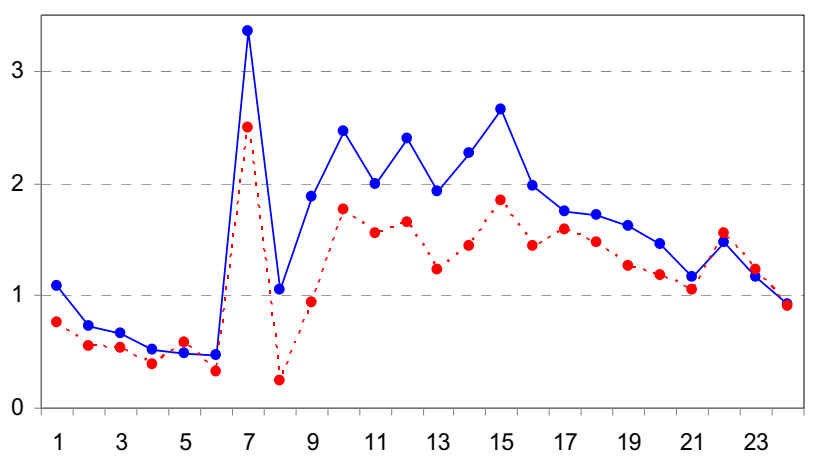

Figure 2: Trends in Average Demand for CT Services ('hour of the day' vs. 'number of arrivals').

Table 3: Modeling the Patient Access Delays.

\begin{tabular}{|c|c|l|}
\hline $\begin{array}{c}\text { Patient } \\
\text { Class }\end{array}$ & $\begin{array}{c}\text { Oral } \\
\text { Contrast }\end{array}$ & $\begin{array}{c}\text { Patient Access Delay } \\
\text { (minutes) }\end{array}$ \\
\hline \multirow{2}{*}{$\begin{array}{c}\text { Emergency } \\
(\mathrm{ED})\end{array}$} & $\mathrm{YES}$ & $44+254 * \operatorname{BETA}(2.6,2.85)$ \\
\cline { 2 - 3 } & $\mathrm{NO}$ & $3+127 * \operatorname{BETA}(0.755,1.88)$ \\
\hline \multirow{2}{*}{$\begin{array}{c}\text { Inpatients } \\
(\mathrm{IP})\end{array}$} & $\mathrm{YES}$ & $15+\mathrm{WEIB}(217,1.65)$ \\
\cline { 2 - 3 } & $\mathrm{NO}$ & $15+528 * \mathrm{BETA}(0.637,1.74)$ \\
\hline \multirow{2}{*}{$\begin{array}{c}\text { Outpatients } \\
(\mathrm{OP})\end{array}$} & $\mathrm{YES}$ & $0.999+\operatorname{EXPO}(94.8)$ \\
\cline { 2 - 3 } & $\mathrm{NO}$ & $0.999+\mathrm{EXPO}(33.8)$ \\
\hline
\end{tabular}

\section{BASELINE MODELING AND SIMULATION}

With all the essential data inputs derived, a simulation model of the CT examination process was developed using Rockwell Arena ${ }^{\circledR}$ software. This entailed an emulation of the patient flow logic (illustrated in Figure 1) within the simulation software. In the model, patient arrivals were controlled by schedules corresponding to the established arrival patterns while the patient access delays 
and service times were represented by their respective probability density functions. The model incorporated the baseline staffing patterns as well as the operational characteristic of preferring M1 over M2 (as elaborated in Section 4). The model was developed with the premise that:

1) There was no significant difference in the time required to perform a $\mathrm{CT}$ examination with or without contrast, since preparatory tasks were done outside the examination area.

2) The time taken to conduct a CT procedure was independent of the day of the week and the operating technologist.

3) The technologists' productive time consisted of all the contact time with the patient as well as any additional time taken to handle the associated paperwork.

4) A replication length of one week (seven days) was sufficient to closely emulate the true operating characteristics of the CT-scan facility.

\subsection{Model Verification and Validation}

The completed baseline model was logically verified and ascertained to bear the intended patient flow logic. It was then configured with a replication length of one week and executed for 30 replications, after which its key performance measures were collected and analyzed. The weekly patient throughput and the 'examination process delays' exhibited by the simulation results were then used to check for the validity of the baseline model. Paired t-tests were utilized to compare the simulated patient throughput (weekly averages) with the actual values, revealing that there was no significant difference. Discussions were then held with the process experts to evaluate the examination process delays portrayed by the simulation results. Once again, the trends observed in the examination process delays were confirmed to be typical of the CT scan processes.

\subsection{Baseline Performance Measures}

The simulation results suggested that resource capacity was not a constraint for the CT processes. This was inferred from the observation that the average weekly utilization for the various resources was less than $50 \%$ (as illustrated in Table 4). Therefore, focus was shifted to the waiting characteristics experienced by patients visiting the $\mathrm{CT}$ area. Specific interest was vested on the examination process delays, since they directly impacted the quality of care perceived by the patient (timeliness), and they were controllable. The baseline results indicated that patients would experience an average delay of 11 minutes, with an average queue length of less than one. These results lacked the depth of information that was required to facilitate a better understanding of the waits experienced by patients seeking $\mathrm{CT}$ procedures, and how this impacted their satisfaction.

Table 4: Baseline Resource Utilization Levels.

\begin{tabular}{|l|c|l|}
\hline \multicolumn{1}{|c|}{ Resource Type } & Capacity & $\begin{array}{c}\text { Average } \\
\text { Utilization }\end{array}$ \\
\hline 64 slice scanner & 1 & $30.5 \%$ \\
\hline 4 slice scanner & 1 & $22.9 \%$ \\
\hline Day shift tech. (weekday) & 3 & $24.7 \%$ \\
\hline Day shift tech. (weekend) & 1 & $42.1 \%$ \\
\hline Evening Shift tech. & 1 & $36.4 \%$ \\
\hline Night shift tech. & 1 & $25.7 \%$ \\
\hline
\end{tabular}

Detailed analysis showed that the delays exhibited different patterns throughout the day for each of the three patient categories. For instance, Figure 3 illustrates the delay patterns experienced by ED patients seeking CT procedures. As shown, the hourly mean and median delays (unfilled and filled circular dots) have an average of zero minutes for most parts of the day with the exception of the peak periods ( $6 \mathrm{pm}$ to $2 \mathrm{am}$ ) when they vary significantly. Generally, there was a significantly higher volume of arrivals to the emergency department after $4 \mathrm{pm}$, leading to an increased demand for CT-scan procedures. This implied that some patients were likely to experience long waits and probably get dissatisfied by the "seemingly efficient" examination process.

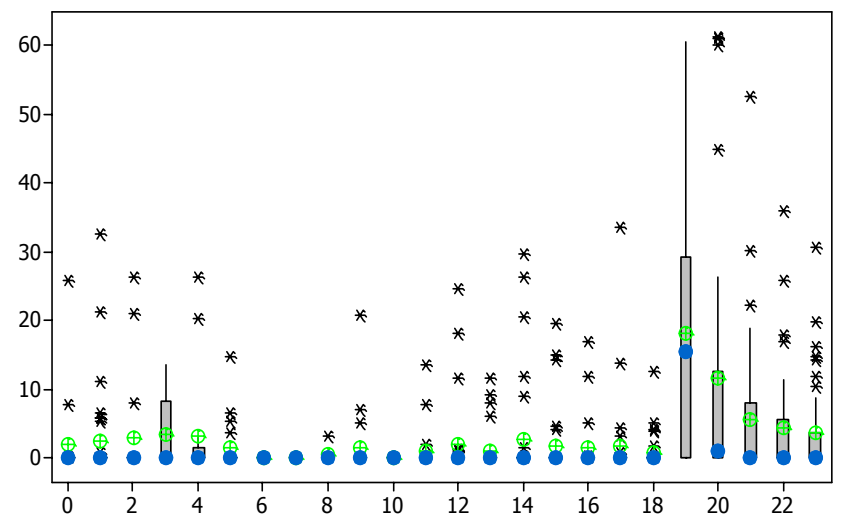

Figure 3: Wait Time Trends for ED Patients (baseline).

To quantify this observation, it was assumed that patients would become impatient and consequently dissatisfied if they experienced waits longer than ten minutes. Based on this premise, the process defects (unsatisfied patients) were computed for each patient category and utilized to evaluate the quality of service as perceived by patients seeking the CT-scan services. This was achieved by using 'Six-Sigma' concepts to compute the Defects Per Million Opportunities (DPMO) for each patient category. 'Sigma levels' (the variation from perfection based on the 
amount of defects) were then computed to facilitate the evaluation of process performance based on opportunities and defects. The resulting defect opportunities and sigmalevels for the baseline model are shown in Table 5. Essentially, higher sigma levels are preferable as they signify that a process is associated with fewer defects. Perfection (zero defect level) is normally achieved beyond the six sigma level.

Table 5: Defect Rates for the Baseline Model.

\begin{tabular}{|c|c|c|c|}
\hline $\begin{array}{c}\text { Simulation } \\
\text { Options }\end{array}$ & & \multicolumn{2}{|c|}{ Current Volume } \\
\hline & & Sigma & DPMO \\
\hline \multirow{2}{*}{$\begin{array}{c}\text { BASELINE } \\
\text { LAYOUT }\end{array}$} & ED & 2.85 & 88,785 \\
\cline { 2 - 4 } & IP & 2.85 & 89,474 \\
\cline { 2 - 4 } & OP & 3.45 & 25,424 \\
\hline
\end{tabular}

\section{WHAT-IF ANALYSIS}

As mentioned above, this simulation study was tasked with facilitating decision making regarding the capacity and design option (location on facility layout) that would be adopted for the CT scan facilities with the aim of upholding high levels of patient satisfaction. Initial stakeholder suggestions included (1) the creation of an additional imaging unit, and (2) the adoption of decentralized operations with different imaging units dedicated to serving different patient categories. An ideal alternative was expected to support improved patient satisfaction, even with the expected growths in the annual throughput (growth of 25 to $50 \%$ in CT procedures). To test these factors, three study scenarios were developed and used as part of the simulation experiments. These are delineated below.

1) Scenario I: Considered an increase in the capacity of the imaging units by one. It featured a decentralized model for the units, each being dedicated to serving a single patient category (IP, OP or ED). These would be located within the established patient-flow pathways, thereby making it infeasible to utilize a single imaging unit to service more than one patient category. Thus, patient queues would potentially form at each of the three units.

2) Scenario II: Also considered an increase in the capacity of the imaging units by one. The three units would form a centralized model whereby all the three patient categories would be serviced in a FIFO manner, with all biopsy procedures being performed in a specific unit.

3) Scenario III: Was a replication of the baseline scenario, albeit with a significantly altered staff- ing pattern. It featured two imaging units utilized interchangeably, with all biopsies being done in a specified unit. The staffing pattern utilized was modified to closely match the established patient arrival behavior.

For each of the three scenarios, it was assumed that 64-slice CT-scanners would be utilized, thereby supporting the premise that all imaging units would be equally preferable. The scenarios also featured staffing patterns that were considerably different from the baseline staffing. These were generated based on the hourly variations observed in the arrival patterns of the various patient categories. These scenarios were developed and analyzed considering a $25 \%$ and $50 \%$ increase in patient volumes, corresponding to the limits of the expected growth in annual patient volume. For each scenario, the average utilization levels as well as the associated queuing characteristics were subjected to analysis. Once again, focus was placed on understanding the trends exhibited by the examination process delays of the various patient categories. For this purpose, the analysis technique introduced in Section 6.2 was utilized to compute the defect opportunities associated with each design alternative. This information is summarized in Table 6.

Table 6: Defect Rates for the Alternative Scenarios.

\begin{tabular}{|c|c|c|r|r|r|}
\hline $\begin{array}{c}\text { Simulation } \\
\text { Options }\end{array}$ & & \multicolumn{2}{|c|}{$\begin{array}{c}\mathbf{2 5 \%} \text { Increase } \\
\text { in Visits }\end{array}$} & \multicolumn{2}{c|}{$\begin{array}{c}\mathbf{5 0 \%} \text { Increase } \\
\text { in Visits }\end{array}$} \\
\hline & & Sigma & DPMO & Sigma & DPMO \\
\hline \multirow{3}{*}{$\begin{array}{c}\text { SCENARIO } \\
\text { I }\end{array}$} & ED & 2.75 & 101,759 & 2.50 & 154,472 \\
\cline { 2 - 6 } & IP & 2.25 & 219,400 & 2.00 & 308,328 \\
\cline { 2 - 6 } & OP & 2.35 & 196,141 & 2.20 & 241,692 \\
\hline \multirow{3}{*}{$\begin{array}{c}\text { SCENARIO } \\
\text { II }\end{array}$} & ED & 3.40 & 29,948 & 3.35 & 30,851 \\
\cline { 2 - 6 } & IP & 3.25 & 40,931 & 3.00 & 64,472 \\
\cline { 2 - 6 } & OP & 4.15 & 3,788 & 3.25 & 39,437 \\
\hline \multirow{3}{*}{$\begin{array}{c}\text { SCENARIO } \\
\text { III }\end{array}$} & ED & 3.40 & 27,578 & 3.30 & 37,736 \\
\cline { 2 - 6 } & IP & 3.30 & 34,367 & 3.10 & 57,241 \\
\cline { 2 - 6 } & OP & 3.65 & 15,209 & 3.30 & 34,591 \\
\hline \multicolumn{2}{|c|}{} & \multicolumn{4}{|c}{} \\
\hline
\end{tabular}

The resource utilization levels (CT-scan units and technologists) associated with all the scenarios were noted to be less than $50 \%$, even at the elevated throughput levels. This confirmed that capacity was not anticipated to pose operational challenges to the $\mathrm{CT}$ examination processes (at present and in the near future). It was though observed that the defect opportunities would vary significantly for the different scenarios depending on the throughput level. As illustrated in Table 6, Scenarios II and III were observed to be relatively comparable in terms of their defect opportunities. Compared to Scenario I and the baseline model, Scenarios II and III would be expected to produce fewer defects, corresponding to 
lower incidences of patient delays and subsequent low levels of dissatisfaction.

Despite the close functional similarities anticipated for Scenarios II and III, Scenario III utilized fewer imaging units and would eventually require a lower number of man-hours (paid personnel hours) per week. As illustrated in Table 7, Scenario III would require two imaging units (similar to the baseline) and a total of 336 manhours per week. This suggested that Scenario III, which featured a slight modification of the existing system, would be the "ideal" design alternative. It would facilitate the realization of higher levels of patient satisfaction by utilizing the existing capacity of the imaging units

Table 7: Weekly Staffing for the Alternative Scenarios.

\begin{tabular}{|r|c|c|}
\hline \multicolumn{1}{c|}{ Configuration } & $\begin{array}{c}\text { Weekly Scheduled } \\
\text { Man-Hours }\end{array}$ & $\begin{array}{c}\text { Imaging } \\
\text { Units }\end{array}$ \\
\hline BASELINE & 296 & 2 \\
\hline SCENARIO I & 392 & 3 \\
\hline SCENARIO II & 304 & 3 \\
\hline SCENARIO III & 336 & 2 \\
\hline
\end{tabular}

\section{CONCLUSIONS}

Based on the outcomes of this simulation study, recommendations were made to streamline the CT-scan processes and review the associated staffing levels to ensure that they matched the anticipated patient arrival patterns. To facilitate the facilities design for the new hospital, it was recommended that a centralized CT-scan model be adopted (depicted in Scenarios II and III). Specifically, Scenario III was recommended as the 'best design alternative' since it featured improved patient flow dynamics (fewer defect opportunities). Besides, it was associated with financial benefits as it would not necessitate capital spending on an additional imaging unit.

The centralized design was expected to facilitate 'level loading' (balanced workload) between the CT-scan units, a feature that was not possible with the decentralized design. This meant that constraints in patient flow could easily be evened out in the centralized design, whereas similar constraints would possibly result to bottlenecks in the decentralized design. Therefore, the centralized design was expected to exhibit a more streamlined patient flow behavior. This was also associated with reduced wait times, which consequently resulted to improved patient experience. Among other things, this option enhanced the timeliness dimension of quality that is sought for by many healthcare providers.

\section{REFERENCES}

Davis W. 1999. Simulation: technologies in the new millennium, In Proceedings of the 1999 Winter Simulation Conference, ed. P. Farrington, H. Nembhard, D. Sturrock, and G. Evans, 141-147. Phoenix, Arizona.

Jun, J., S. Jacobson, and J. Swisher. 1999. Application of discrete event simulation in health care clinics: a survey. The Journal of Operational Research Society, 50 (2):109-123.

Law, A. and D. Kelton. 2003. Simulation modeling and analysis, $3^{\text {rd }}$ ed., New Delhi, India: McGraw-Hill.

Ramakrishnan, S., A. Courtney, K. Srihari, and F. Emick. 2004. A study of the CT scan area of a healthcare provider, In Proceedings of the 2004 Winter Simulation Conference, ed. R. G. Ingalls, M. D. Rossetti, J. S. Smith, and B. A. Peters, 2025-2031. Washington, D.C.

Sg2. 2007. Building Tomorrow's Imaging Facilities, Available via <http://www.sg2.com> [accessed March 10, 2007].

\section{AUTHOR BIOGRAPHIES}

JOSHUA BOSIRE is a Masters student in the Thomas J. Watson School of Engineering and Applied Science at the State University of New York at Binghamton, New York. His current research involves the analysis and improvement of healthcare delivery systems using Industrial and Systems Engineering techniques.

SHENGYONG WANG is a Research Assistant Professor in the Department of Systems Science and Industrial Engineering at the Thomas J. Watson School of Engineering and Applied Science at the State University of New York at Binghamton, New York. His research areas include healthcare engineering, systems modeling and simulation, and discrete event systems.

TEJAS GANDHI is the Director of the Management Engineering Department at the Virtua Health, a multi-facility provider in South New Jersey. He facilitates the use of applied research in executive decision making while working with all levels of the organization to improve performance and optimize processes.

KRISHNASWAMI SRIHARI is a Distinguished Professor and the Chair of the Department of Systems Science and Industrial Engineering at the Thomas J. Watson School of Engineering and Applied Science of the State University of New York at Binghamton, New York. 